\title{
Evaluation of a Microplasma Source Based on a Stripline Split-Ring Resonator
}

\author{
M Berglund ${ }^{1}$, M Grudén ${ }^{2}, \mathrm{G}$ Thornell $^{1}$ and A \\ Persson $^{1}$ \\ 1 Ångström Space Technology Centre, Dept. of Engineering Sciences, \\ Uppsala University Box 534 SE-75121, Uppsala, Sweden \\ 2 Solid State Electronics, Dept. of Engineering Sciences, Uppsala \\ University Box 534 SE-75121, Uppsala, Sweden \\ E-mail: martin.berglund@angstrom.uu.se
}

This document is the accepted manuscript version of a published work that appeared in final form in Plasma Sources Science and Technology, after peer review and technical editing by the publisher. To access the final edited and published work see:

http://dx.doi.org/10.1088/0963-0252/22/5/055017

\begin{abstract}
In this paper, a stripline split-ring resonator microwave-induced plasma source, aimed for integration in complex systems, is presented and compared with a traditional microstrip design. Devices based on the two designs are evaluated using a plasma breakdown test setup for measuring the power required to ignite plasmas at different pressures. Moreover, the radiation efficiency of the devices is investigated with a Wheeler cap, and their electromagnetic compatibility is investigated in a variable electrical environment emulating an application. Finally, the basic properties of the plasma in the two designs are investigated in terms of electron temperature, plasma potential, and ion density. The study shows that, with a minor increase in plasma ignition power, the stripline design provides a more isolated and easy-to-integrate alternative to the conventional microstrip design. Moreover, the stripline devices showed a decreased antenna efficiency as compared to their microstrip counterparts, which is beneficial for plasma sources. Furthermore, the investigated stripline devices exhibited virtually no frequency shift in a varying electromagnetic environment, whereas the resonance frequency of their microstrip counterparts shifted up to $17.5 \%$. With regards to the plasma parameters, the different designs showed only minor differences in electron temperature, whereas the ion density was higher with the stripline design.
\end{abstract}

Keywords: Split-ring resonator, Microwave plasma, Microstrip, Stripline, Plasma, Wheeler cap, Langmuir probe

Submitted to: Plasma Sources Sci. Technol. 


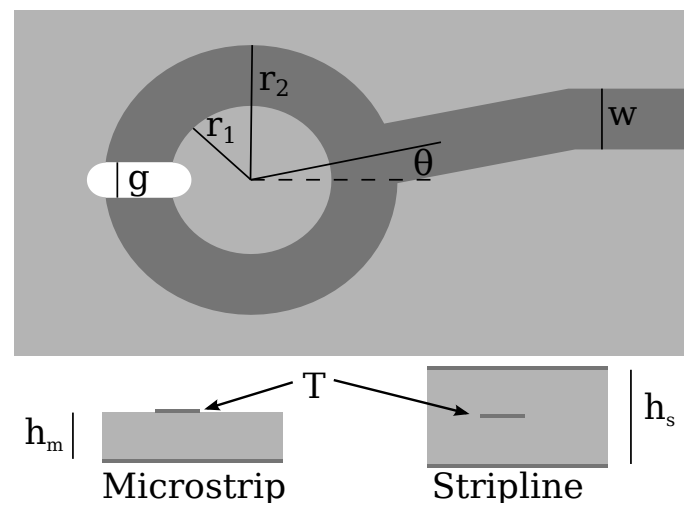

Figure 1. Schematic top view of a split-ring resonator (top), and cross sections of its two embodiments (bottom). Light gray represents the dielectric, and dark gray the conductor material.

\section{Introduction}

Microstrip split-ring resonator microwave-induced plasma sources (MSRR-MIPs) [1, 2, 3, 4, 5, 6], have been proposed for integration in a wide variety of portable devices aimed for, e.g., chemical analysis systems [7, 8], ion sources [9], sterilization or activation of substances [10, 11]. Reasons for this are: their low power consumption, power efficiency, design simplicity, mechanical robustness, high density non-thermal plasma, long lifetime, and ability to operate at atmospheric pressure [4].

When miniaturizing whole systems, the ability to integrate subsystems in close proximity without electromagnetic interference between the components is important. A microstrip is a radio frequency $(\mathrm{RF})$ transmission line consisting of a dielectric sandwiched between a ground plane and a flat strip of metal, figure 1. An MSRR-MIP is designed to be at resonance when the microstrip has a ground plane beneath its substrate and free space above, figure 1 and 2 . This design imposes restrictions on what other systems can be built on top of the microstrip, since a change of the dielectric properties of the surrounding will result in a change of the characteristic impedance of the microstrip itself.

An alternative to the microstrip is to use a type of RF transmission line called a stripline. A stripline works similarly to a microstrip. It consists of a flat strip of metal sandwiched between two dielectric layers, each with its own ground plane, figure 1. A stripline provides good electromagnetic isolation from the ambient with respect to, e.g., varying conductivity and relative permittivity.

In order to facilitate easy integration of a miniaturized plasma source in a complex system, this work presents and evaluates a stripline split-ring resonator (SSRR) concept in terms of plasma ignition power, plasma parameters, radiation efficiency, electromagnetic compatibility (EMC) and shielding, by comparing it with its microstrip equivalent.

\section{Theory}

The design of the split-ring resonators relies on the concepts and theories presented in [4]. A split-ring resonator is basically a curved dipole, where the two ends of the dipole create a small gap over which an electric field can be created. In this context, a dipole is a half-wavelength 


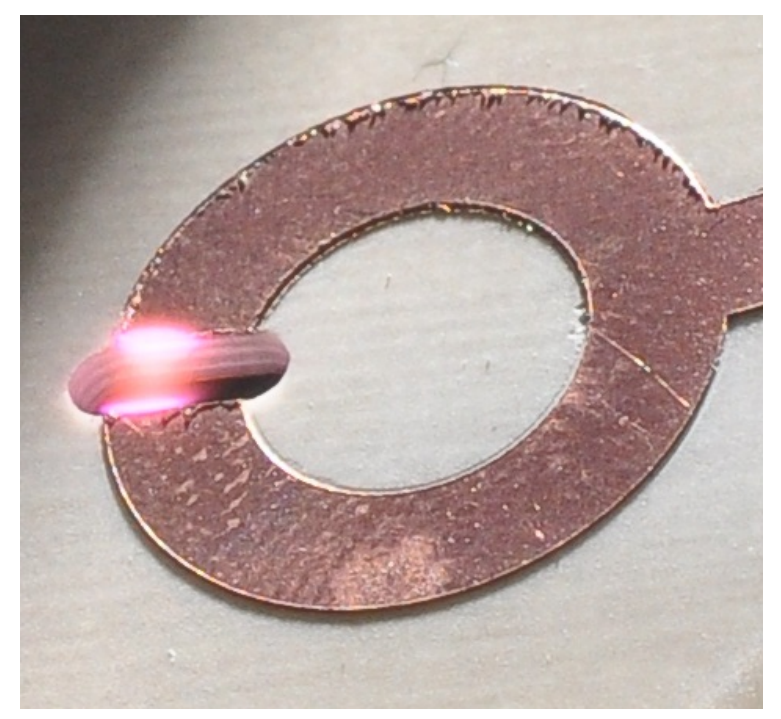

Figure 2. MSRR with plasma ignited in air at a pressure of 3 Torr. The gap of the device is $2 \mathrm{~mm}$ wide. Raking light makes the edges of the split-ring look rough.

microwave resonator, often used as an antenna. In order to effectively feed the dipole with power, the input impedance of the dipole should match the impedance of the transmission line that supplies it. Depending on the quality factor, Q, of the microstrip (or stripline), the dipole must be fed asymmetrically, where higher $\mathrm{Q}$ requires a less asymmetrical feeding and vice versa.

With a relatively low input power, a large potential can be created over the gap [4]. When fed with resonant RF power, a standing wave is created in a dipole, or SRR, where the current is zero at the endpoints and at its maximum at the middle of the strip. Accordingly, the electric potential is at its maximum at the endpoints and zero at the geometrical midpoint of the strip. Moreover, the electric field at the two endpoints is $180^{\circ}$ out of phase, i.e., when, for instance, the standing wave has its maximum at one end and its minimum at the other. This, together with the spatial proximity of the endpoints, serves to amplify the electric field over the gap by orders of magnitude.

The electric field in a microstrip, as well as in a stripline, is mostly confined to the dielectric material between the strip and the ground plane/planes. In the gap, the electric field from one end of the split ring interacts with that of the other end, and the electric field is no longer confined to the dielectric, but is concentrated to the region between the two ends. It is one of the key features of the SRR, that most of the electric field is concentrated to the gap[1]. It should be noted that no DC potential is applied to the plasma, thus minimizing energy lost to moving ions.

Unique to the work presented here is that the dielectric and the ground plane in the gap region have been removed, figure 1. In the stripline design, this was done in order to make the plasma accessible from the outside, but also to reduce losses in the dielectric and to allow the plasma to extend into the plane of the SRR. Other minor changes were to increase the operating frequency to between 2.6 and $2.8 \mathrm{GHz}$ and to make the gap larger. A larger gap is not optimal, since the electric field strength in the gap decreases, but it provides integrability. For example, the open gap can allow for gases to pass through the plasma in a controlled fashion, enabling applications in a wide variety of fluidic systems. 
Table 1. Dimensions (cf. figure 1) and design properties of evaluated devices. These values are all theoretical values calculated using LineCalc (in ADS 2011.10).

\begin{tabular}{llllllll}
\hline ID & Type & $\mathrm{Z}_{0}(\Omega)$ & $r_{1}(\mathrm{~mm})$ & $r_{2}(\mathrm{~mm})$ & $\theta\left(^{\circ}\right)$ & $w(\mathrm{~mm})$ & $Q_{t}$ \\
\hline $50 \mathrm{~S}$ & SSRR & 50 & 4.5 & 6.1 & 12.2 & 1.7 & 140 \\
$50 \mathrm{M}$ & MSRR & 50 & 4.2 & 7.6 & 11.7 & 3.4 & 152 \\
$75 \mathrm{~S}$ & SSRR & 75 & 5.0 & 5.6 & 10.9 & 1.7 & 117 \\
$75 \mathrm{M}$ & MSRR & 75 & 5.3 & 6.9 & 10.2 & 3.9 & 133 \\
\hline
\end{tabular}

\section{Materials and methods}

\subsection{Devices}

In order to compare the microstrip and stripline concepts, four different devices were designed and manufactured. The geometric parameters of each device are given in table 1 and defined in figure 1. Two designs were microstrips with characteristic impedances, $Z_{0}$, of 50 and $75 \Omega$, and two were striplines with corresponding impedances. All of the designs had a $2 \mathrm{~mm}$ wide gap.

Two devices, one stripline and one microstrip with $Z_{0}$ of $50 \Omega$, were equipped with Langmuir probes, aligned perpendicularly to the SRR electrodes, in the plane of the resonator structure. One of the two Langmuir probes was connected to ground. The probes consist of about $1 \mathrm{~mm}$ long gold bond wires with a diameter of $25 \mu \mathrm{m}$.

Although the characteristic impedances of the SRRs varied, the input impedance, $Z_{i n}$, of each device was set to $50 \Omega$ by adjusting the feeding angle of the ring. Each SRR was fed with a microstrip or stripline with a characteristic impedance, $Z_{0}$, of $50 \Omega$ at the offset angle, $\theta$, determined by the dipole $\mathrm{Q}$ factor and the input impedance, $Z_{i n}$, of $50 \Omega$. The offset angle was calculated accordingly to

$$
\cos \theta=1-\frac{Z_{\text {in }} \pi}{Z_{0} Q_{t}}
$$

where $Q_{t}$ is the theoretical quality factor of the microstrip or stripline waveguide [4].

The material for the devices was a RO4003C printed circuit board, from Rogers Corporation. This laminate's dielectric layer was made of a glass-fibre-reinforced ceramic composite, having a thickness of $1.52 \mathrm{~mm}$, a dielectric constant, $\varepsilon_{r}$, of 3.38, and a dissipation factor, $\tan \delta$, of 0.0022 . On both sides was a $70 \mu \mathrm{m}$ thick copper cladding.

The shape and size of each device were calculated and designed using LineCalc in Advanced Design System 2011.10 (ADS) from Agilent Technologies, aiming for resonance at 2.6 GHz, table 1 . The resulting CAD files were used to mill the devices from the laminate, using an LPKF ProtoMat S100 PCB plotter. The stripline devices consist of one top and one bottom part, where the bottom part comprises the ring, and the top part has a countersink made with a rub-out milling tool. The countersink was necessary for the laminates to fit on top of each other.

The two stripline source parts were glued together, using a thin layer of epoxy. Finally, SMA connectors (end-launch SubMiniature version A) were soldered to the feeding strip of the devices, with the central conductor connected to the feeding strip, and the shield connected to the groundplane(s). 


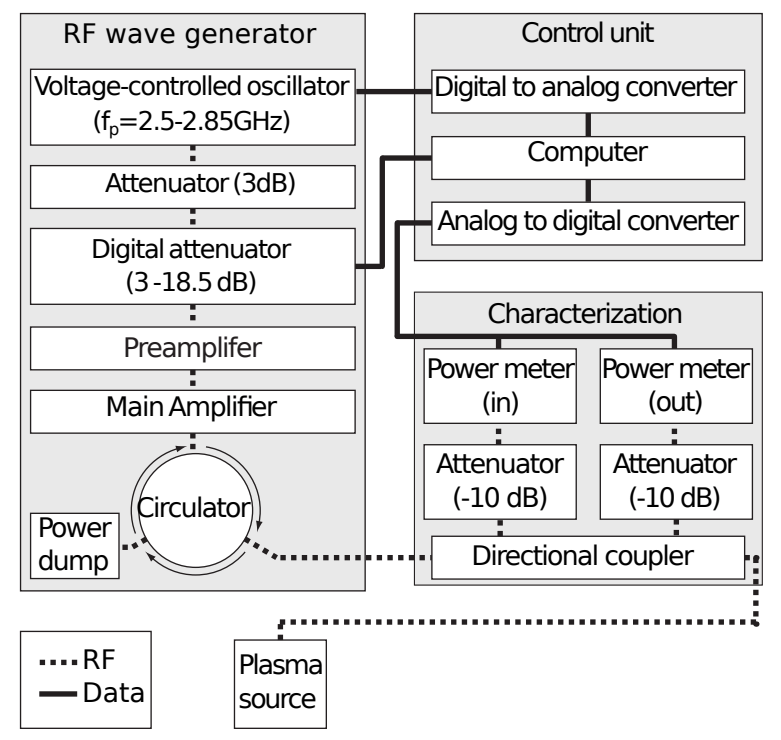

Figure 3. Schematic of RF power supply and measurement setup.

\subsection{Setup}

In order to verify the power required to ignite a plasma in each device, an RF power supply and a vacuum chamber were used. The RF power supply, figure 3, consisted of a chain of circuits starting with a voltage-controlled oscillator (VCO), Mini-Circuits ZX95-3000W-S + , for controlling the frequency of the RF sine wave. After the VCO, a fixed $3 \mathrm{~dB}$ attenuator, followed by a digitally controlled attenuator, Mini-Circuits ZX76-15R5-S+, with a range of 3-18.5 dB, were employed to control the power going into the device under test (DUT). Then, two amplifiers in series, firstly a pre-amplifier, Mini-Circuits ZRL-3500+, and secondly a main amplifier, Cree CMPA 2560025F, were used to amplify the RF wave to a maximum power of $18 \mathrm{~W}$ in a range from 2.5 to $2.85 \mathrm{GHz}$. The amplifiers were followed by a circulator, MA-COM MAFR-000050-5S4C1T, connected to a directional coupler, Mini-Circuits ZABDC20-322H-S+. Finally, the directional couplers were connected to the DUT. The power transmitted to, and reflected from, the DUT was measured by two power meters, Mini-Circuits ZX47-40-S+, connected to the directional coupler, and logged by a computer. The reflected power was dumped into a termination. The entire RF setup was interconnected by $50 \Omega$ SMA interfaces and coaxial cables.

The vacuum chamber had a base pressure of about 1.5 Torr. The pressure was controlled by a needle valve, and the RF power was delivered to the DUT through an SMA feed-through in the chamber wall.

The Langmuir probe measurements were carried out with a TTi QL355TP power supply and an Agilent 34972A DAQ with an Agilent 34901A 20 Channel Multiplexer connected in series with the two Langmuir probes. The voltage source was able to set the voltage over the two probes between -70 and $70 \mathrm{~V}$ with three decimal digits, and the current through the plasma was measured with 6.5 digit resolution. The RF power to the plasma was set sufficiently high to keep the Langmuir probes completely immersed regardless of the probe bias voltage, as confirmed by visual inspection. 


\subsection{Measurements}

The first characterization entailed measuring the $S_{11}$ of the devices in free space, using a network analyzer, Agilent Technologies E8364B, in order to determine how well the quality factor and the resonance frequency matched the design values. Moreover, theoretical calculations of the voltage $V_{g}$ across the gap were performed using

$$
V_{g}=4 \sqrt{\frac{Z_{0} Q}{\pi}} \sqrt{P_{\mathrm{in}}},
$$

where $Z_{0}$ is the characteristic impedance of the SRR, $Q$ is the measured quality factor, and $P_{\text {in }}$ is the accepted power of the device [4].

The power required to ignite each plasma source at a certain pressure was measured by stabilizing a fixed air pressure in the chamber, and feeding the DUT with two 1-s pulses with a 1-s separation. After a 10-s cool-down period, the process was repeated with the power increased by $0.5 \mathrm{~dB}$, until the reflected power jumped from about $-12 \mathrm{~dB}$ to between -3 and $-6 \mathrm{~dB}$, indicating

a plasma ignition. The first occurrence of a plasma ignition was marked as the ignition power for that particular pressure, and a new pressure was set and the process repeated, starting at a low power. Plasma ignition, figure 2, could also be confirmed visually through a sight glass on the vacuum chamber.

In order to test the EMC of the devices, they were placed between two $200 \times 200 \mathrm{~mm}$ singlesided FR4 PCB plates separated by 0,15 or $32 \mathrm{~mm}$, where $0 \mathrm{~mm}$ corresponded to the device being in contact with the plates. The reflection coefficient, $S_{11}$, was measured using the network analyzer. The metalized sides of the FR4 PCBs were facing outwards from the device in order not to short-circuit to the microstrip. However, this was not a risk in the case of the stripline devices. A measurement with the plates removed was also performed. The output from each measurement was $S_{11}$ data between 2 and $3 \mathrm{GHz}$, including the amplitude and phase of the reflected wave.

In order to characterize the power radiation of each device, the Wheeler cap method, for measuring the efficiency of antennas, was used $[12,13,14]$. This method is based on reflecting all of the device's radiation while causing minimal disturbance to the near field. This was achieved by inserting the device into a conductive cylinder with a radius of $\sim \lambda / 2 \pi(19 \mathrm{~mm}$ at $2.6 \mathrm{GHz})$, i.e., with its wall at the border between the near and far field. The cylinder used in this experiment was made of stainless steel, and had an inner radius of $22.5 \mathrm{~mm}$ and a height of $62 \mathrm{~mm}$. One at a time, the devices were placed inside the cylinder and a $S_{11}$ measurement between 2 and $3 \mathrm{GHz}$ was performed using the network analyzer. The devices were placed with the SRR close to the center, and the SMA cable going out the bottom of the cylinder. Subsequently, the antenna efficiency, $\eta$, was calculated using

$$
\eta=1-\frac{\left(1-S_{11 \mathrm{FS}}\right)\left(1+S_{11 \mathrm{WC}}\right)}{\left(1+S_{11 \mathrm{FS}}\right)\left(1-S_{11 \mathrm{WC}}\right)},
$$

where $S_{11 \mathrm{FS}}$ and $S_{11 \mathrm{WC}}$ are the closest real points to the resonance frequency on the reflection coefficient curve [14]. FS and WC denote free space and Wheeler cap, respectively.

The plasma parameters of the two designs were investigated using the two devices equipped with Langmuir probes. The plasma was ignited and maintained at a constant air pressure of 5.76 Torr, with a constant power of $4.47 \mathrm{~W}$. A voltage of random amplitude ranging between -70 and $70 \mathrm{~V}$, was applied to the Langmuir probe not connected to the ground, while measuring the current. Random voltages were used to avoid systematic errors from electrostatic charging of the electrodes. From the resulting I-V curve, the ion density, the electron temperature and the plasma 


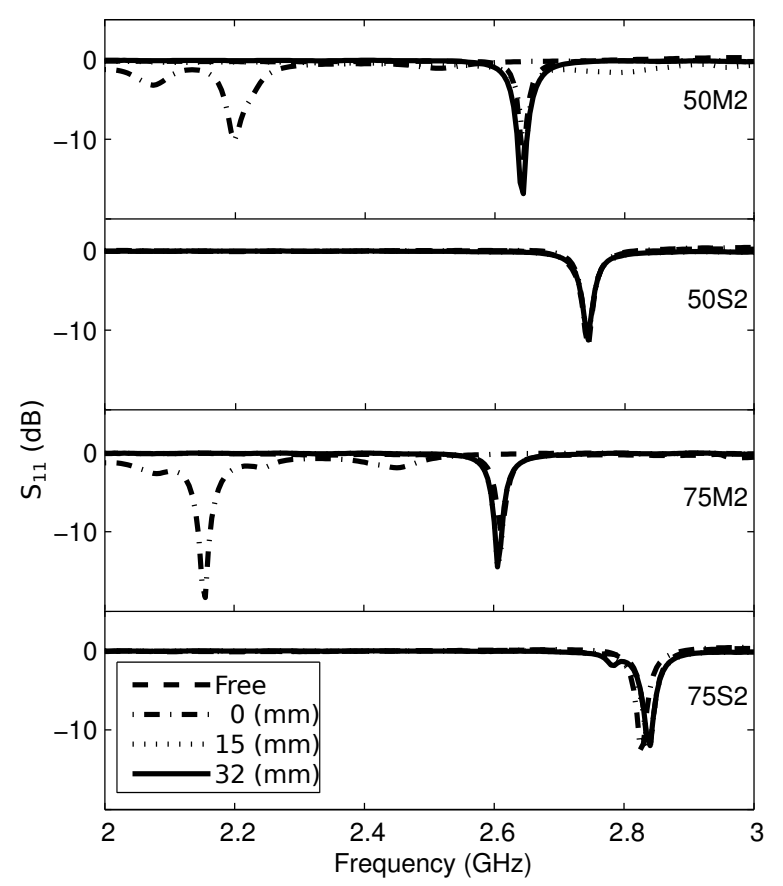

Figure 4. $S_{11}$ of the devices while operated between two metalized FR4 PCBs at different separations.

Table 2. Measured electrical parameters of the devices. $\mathrm{V}_{g}$ is the peak-to-peak ignition voltage when the plasma source is fed with $1 \mathrm{~W}$ of RF power, calculated from the measured $\mathrm{Q}$ and the theoretical characteristic impedance, using equation (2).

\begin{tabular}{clcc} 
Device & $\mathrm{f}_{0}(\mathrm{GHz})$ & $\mathrm{Q}$ & $\mathrm{V}_{\mathrm{g}}(\mathrm{V})$ \\
\hline $50 \mathrm{~S}$ & 2.744 & 73 & 136 \\
$50 \mathrm{M}$ & 2.645 & 89 & 151 \\
$75 \mathrm{~S}$ & 2.838 & 75 & 169 \\
$75 \mathrm{M}$ & 2.611 & 82 & 177 \\
\hline
\end{tabular}

potential could be determined. The probe area used to calculate the ion density was $0.0704 \mathrm{~mm}^{2}$ and $0.1010 \mathrm{~mm}^{2}$ for the $50 \mathrm{M}$ and $50 \mathrm{~S}$ devices, respectively. The length of each probe, subject to manufacturing inaccuracy, was measured using a microscope.

\section{Results}

The quality factor, $Q$, and resonance frequency, $f_{0}$, calculated from the $S_{11}$ measurements, figure 4 , can be seen in table 2, including theoretical calculations of $V_{\mathrm{g}}$ at $1 \mathrm{~W}$ of power, using (2).

Figure 5 shows the power required to ignite the devices at different pressures. Each point shows the lowest power where a plasma discharge occurred for a certain pressure. The maximum power output was limited by the setup.

Figure 4 shows the $S_{11}$ curves of the devices while they are operated between the two metallic 


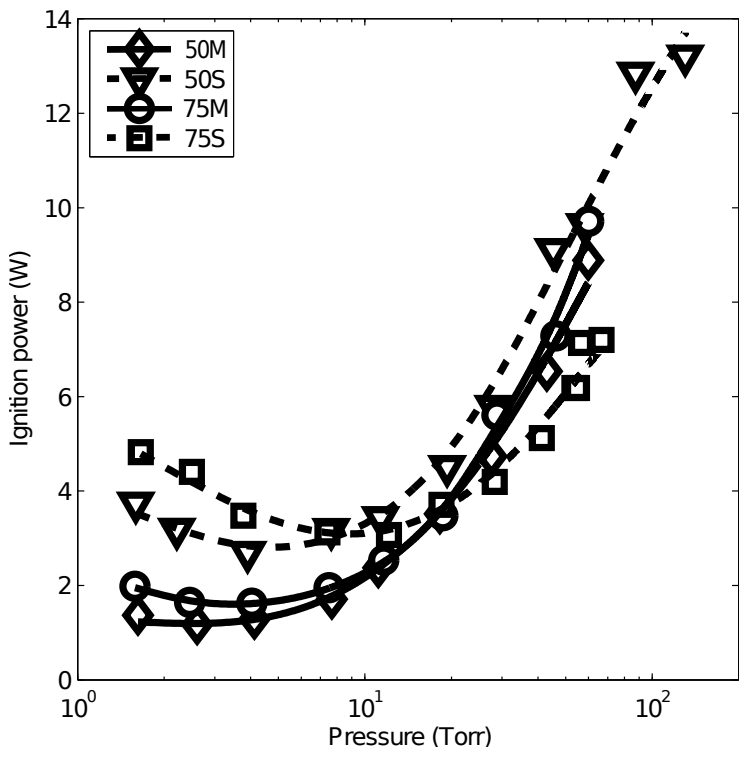

Figure 5. Ignition power versus air pressure.

Table 3. Change in resonance frequency when the device is centered between two metalized FR4 PCBs at different separations.

\begin{tabular}{cccc} 
Device & $0 \mathrm{~mm}$ & $15 \mathrm{~mm}$ & $32 \mathrm{~mm}$ \\
\hline $50 \mathrm{~S}$ & $0.03 \%$ & $0.02 \% 0$ & $0.04 \% 0$ \\
$50 \mathrm{M}$ & $16.8 \%$ & $0.73 \% 0$ & $0.46 \% 0$ \\
$75 \mathrm{~S}$ & $4.14 \% 0$ & $0.12 \% 0$ & $0.06 \% 0$ \\
$75 \mathrm{M}$ & $17.5 \%$ & $1.59 \%$ & $1.81 \% 0$ \\
\hline
\end{tabular}

plates of the FR4 PCBs. As can be seen, the resonance frequency of the microstrip device was drastically shifted as the distance between the metal plates was reduced, whereas the resonance frequency of the stripline device was preserved. The resonance shift of the individual devices are given in table 3.

The results of the Wheeler cap measurements are shown in figure 6. Here, the antenna efficiency, i.e., the RF power radiating out from each device, as calculated with equation (3), is given. As can be seen, significant differences between both the two designs and the two characteristic impedances occurred.

The I-V curves of the two devices equipped with Langmuir probes are shown in figure 7 . From these, the electron temperature, $k_{B} T_{e}$, plasma potential, $V_{p}$, and the ion density, $n_{i}$, were calculated, assuming collisionless, unmagnetized plasma conditions, as described in [15], table 4.

\section{Discussion}

Comparing the properties of the two designs, it can be seen that the resonance frequency of the stripline devices was shifted compared with that of the microstrips and the design goal, table 
Table 4. Plasma parameters calculated from the I-V curves presented in figure 7.

\begin{tabular}{lcc} 
Parameter & $50 \mathrm{M}$ & $50 \mathrm{~S}$ \\
\hline Electron temperature, $k_{B} T_{e}[\mathrm{eV}]$ & 2.83 & 2.78 \\
Plasma potential, $V_{p}[\mathrm{~V}]$ & 19.2 & 23.5 \\
Ion density, $n_{i}\left[\frac{1}{m^{3}}\right]$ & $5.7 \times 10^{17}$ & $1.2 \times 10^{18}$ \\
\hline
\end{tabular}

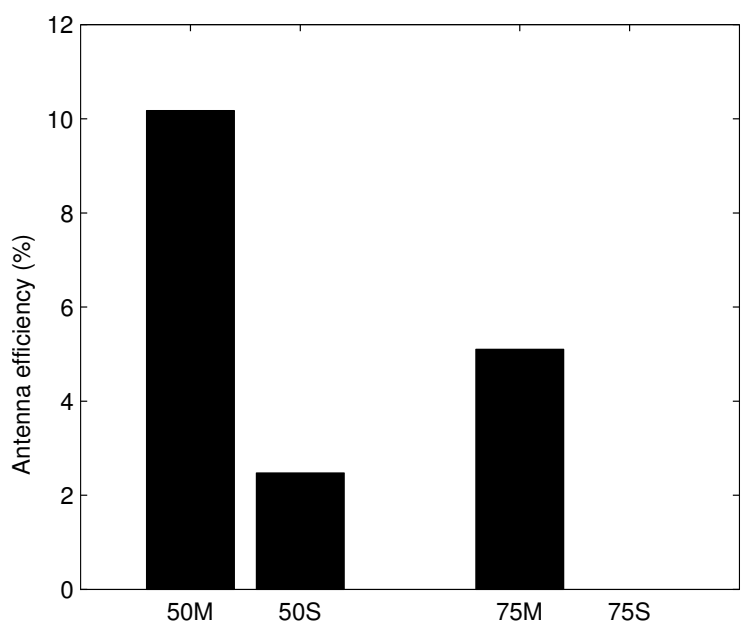

Figure 6. Antenna efficiency of the four devices determined using the Wheeler cap method. The efficiency for the $75 \mathrm{~S}$ device was not resolvable.

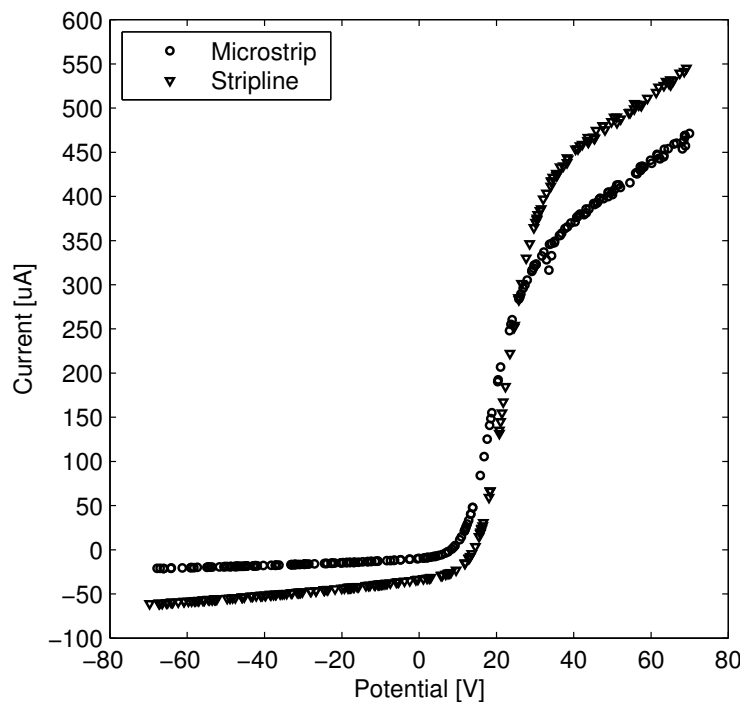

Figure 7. I-V curves for the microstrip (circles) and stripline (triangles) devices equipped with Langmuir probes. The curves were obtained with a $4.5 \mathrm{~W}$ plasma at 5.76 Torr. 
2. This shift can be explained by the approximations and simplifications made when deriving the design parameters. A frequency shift is generally attributed to an additional capacitive or inductive contribution. The small frequency shift of the microstrip sources, as well as part of the shift of the stripline devices, can be attributed to manufacturing issues, e.g., inaccurate width of the leads, voids inside the sandwich structure due to too deep/shallow countersinks, and misalignment of the ground planes.

The measured quality factor, table 2 , is about $50-65 \%$ of the corresponding $Q_{t}$ values, table 1. However, it should be noted that $Q_{t}$ is the calculated quality factor of a waveguide, whereas $Q$ is the quality factor of the entire device. This is probably another symptom of the additional inductive load, or a reduced capacitive load, that caused the frequency shift. The stripline devices had about $90 \%$ the $Q$ value of its microstrip counterpart. This shows that a significant part of the frequency shift for the stripline devices was systematic and probably not caused by fabrication uncertainties. Instead, the frequency shift is assumed to be an artifact from simplifications in the model, e.g, the exclusion of the thin epoxy layer in the stripline.

When comparing the curves in figure 5 , the minimum power required to ignite plasma was higher for the stripline designs $(2.8 \mathrm{~W}$ as compared to $1.2 \mathrm{~W}$ for the $50 \Omega$ devices, and $3.1 \mathrm{~W}$ as compared to $1.6 \mathrm{~W}$ for those with $Z_{0}$ of $75 \Omega$ ). The pressure where the minimum occurred was also shifted upwards for the striplines as compared to the microstrips (from 2.6 to 2.7 Torr for $Z_{0}$ of $50 \Omega$ and from 4.4 to 12.1 Torr for $Z_{0}$ of $75 \Omega$ ).

The stripline plasma sources seem to be more sensitive to gap geometry and the SRR characteristic impedance. The microstrip sources were less affected when the mean free path of the gas was increased with lower pressure, whereas the stripline sources were more rapidly becoming harder to ignite at lower pressures due to the more confined plasma.

The results of the EMC test, figure 4 and table 3, show a significant shift of the resonance frequency downwards when the metal sheets were in close proximity to the microstrip SRR, due to an increased capacitive load on the device. The sripline plasma sources, on the other hand, maintained their resonance frequency. The reflected wave amplitude varied for both the stripline and the microstrip sources, but slightly more for the microstrip. This was probably due to a change in resistivity, following from the blocking of the far field.

The results from the Wheeler cap measurements, figure 6, show very low antenna efficiency for all devices. This is good from a plasma source point of view, since it is preferable that the energy is focused in the gap, and not transformed into radiation. Moreover, a low antenna efficiency minimizes the electromagnetic interference. In this measurement, the stripline sources radiated much less than the corresponding microstrip, highlighting the improved EMC with the stripline concept.

Both the EMC and the Wheeler cap measurements were performed without the presence of plasma. Although the EMC of an ignited device too would be of interest, the EMC before, or rather at, ignition is much more important from an application point of view, since this governs the power required to ignite the plasma. Hence, a stable resonance before ignition enables easy and reliable operation of the device. Moreover, the device becomes much less resonant after ignition, since the plasma affects the dielectric properties of the gap, meaning that an ignited device is less prone to emit electromagnetic radiation and, consequently, is less sensitive to EMC.

The pressure at which the I-V curves, figure 7 , were obtained was at the limit where collisionless sheaths can be assumed, and collisional sheath theory starts to apply. In this region, the models of the sheaths become complicated and difficult to use [16]. However, the probes were completely immersed in the plasma and their radii were small compared to the plasma dimensions, meaning 
that the Bohm ion current, calculated with the collisionless sheath approximation, still should yield an acceptable approximation of the ion density. The electron density, on the other hand, was harder to predict, since the electrons are more susceptible to perturbations from the probes than the less mobile ions. Although to a lesser extent, the calculation of the ion density too was affected by such perturbations. However, these perturbations should affect the measurements on the two designs in a similar way, meaning that a comparative study could be credible, even though the absolute values are less reliable. Moreover, the RF field driving the plasma can be expected to couple to the Langmuir probes, affecting the shape of the I-V curve, and, hence, the calculations of the plasma parameters. However, the density calculations (particularly the calculation of $n_{i}$ ) can be expected to be independent of such RF interference, as long as the ratio between the $f_{0}$ and $p$ is sufficiently high (typically $f_{0} / p>105 \mathrm{~Hz} / \mathrm{Pa}$ ) $[17,18]$. Here, $f_{0} / p$ was in the order of $3.5 \times 10^{6} \mathrm{~Hz} / \mathrm{Pa}$. Still, even at these conditions, RF interference can affect the slope of the I-V curve close to $V_{p}$, and, consequently, the calculation of $T_{e}$ [17]. The amplitude of the RF interference on the probe-to-plasma potential, $V_{R F}$, can be estimated from the second derivative of the probe current:

$$
V_{R F} \simeq \frac{V\left(I_{\min }^{\prime \prime}\right)-V\left(I_{\max }^{\prime \prime}\right)}{2}
$$

where $V$ is the DC voltage applied to the probes [19]. It has been shown that the RF interference has a minor effect on the calculation of $T_{e}$, as long as $V_{R F} /\left(2 k_{B} T_{e} / e\right)<1[17,19]$. In this case, this ratio was 1.12 and 1.11 for the microstrip and stripline devices, respectively. Hence, an RF influence on $T_{e}$ cannot be excluded, and the reported values should therefore be regarded as an upper bound to the actual electron temperature [20].

Comparing the plasma parameters of the two designs, table 4, the stripline design shows almost twice as high ion density as the microstrip. This also resulted in the stripline design having a higher bulk plasma potential than the microstrip. The higher ion density of the stripline SRR probably stems from the fact that the electrical field was more concentrated in this design, given that it is more symmetric from an electromagnetic point of view. It should be noted that a high ion density is an important property in many applications, making the stripline design favorable in yet another perspective.

\section{Conclusions}

With some minor performance losses, most significantly in ignition power at pressures below 20 Torr, the stripline-based SRR microplasma sources have been shown to improve the EMC of a SRR MIP. The microstrip SRRs detuned if metal surfaces were introduced in close proximity to the resonator, due to an additional capacitive load. The stripline sources, on the other hand, were largely unaffected by the introduction of the metal planes. Moreover, microstrip sources radiated 5 and $10 \%$ of the accepted power, whereas the stripline sources radiated only 0 and $2.5 \%$.

The plasma in the two designs was shown to be similar in terms of electron temperature, whereas the stripline design was shown to exhibit more than twice as high ion density as the microstrip. The latter might be advantageous in applications where activation of substances by ionization is needed. However, further studies, employing more elaborate theory, are required in order to fully determine the plasma parameters of a stripline SRR.

The results of this study show that stripline SRRs can be used when integration of SRR MIPs into complex systems is required, e.g., in fluidics or optics, and/or when multiple plasma sources need to be stacked together. 


\section{Acknowledgments}

The authors would like to tank the Swedish National Space Board (SNSB) for making this project possible. The Knut and Alice Wallenberg foundation is acknowledged for funding the laboratory facilities. All contributions from Johan Sundqvist at Ångström Space Technology Centre, Uppsala University, are also very much appreciated.

\section{References}

[1] F. Iza and J.A. Hopwood. Low-power microwave plasma source based on a microstrip split-ring resonator. Plasma Science, IEEE Transactions on, 31(4):782 - 787, aug. 2003.

[2] F. Iza and J.A. Hopwood. Rotational, vibrational, and excitation temperatures of a microwave-frequency microplasma. Plasma Science, IEEE Transactions on, 32(2):498 - 504, april 2004.

[3] J. Hopwood and F. Iza. Ultrahigh frequency microplasmas from 1 pascal to 1 atmosphere. J. Anal. At. Spectrom., 19:1145-1150, 2004.

[4] F Iza and J Hopwood. Split-ring resonator microplasma: microwave model, plasma impedance and power efficiency. Plasma Sources Science and Technology, 14(2):397, 2005.

[5] F. Iza and J.A. Hopwood. Self-organized filaments, striations and other nonuniformities in nonthermal atmospheric microwave excited microdischarges. Plasma Science, IEEE Transactions on, 33(2):306 - 307, apr 2005.

[6] J Hopwood, F Iza, S Coy, and D B Fenner. A microfabricated atmospheric-pressure microplasma source operating in air. Journal of Physics D: Applied Physics, 38(11):1698, 2005.

[7] Vassili Karanassios, Kara Johnson, and Andrea Smith. Micromachined, planar-geometry, atmospheric-pressure, battery-operated microplasma devices (mpds) on chips for analysis of microsamples of liquids, solids, or gases by optical-emission spectrometry. Analytical and Bioanalytical Chemistry, 388:1595-1604, 2007.

[8] Manuela Miclea and Joachim Franzke. Analytical detectors based on microplasma spectrometry. Plasma Chemistry and Plasma Processing, 27:205-224, 2007.

[9] Antje Michels, Sven Tombrink, Wolfgang Vautz, Manuela Miclea, and Joachim Franzke. Spectroscopic characterization of a microplasma used as ionization source for ion mobility spectrometry. Spectrochimica Acta Part B: Atomic Spectroscopy, 62(11):1208 - 1215, 2007.

[10] A. Mizuno. Recent progress and applications of non-thermal plasma. Int. J. Plasma Environ. Sci. Technol., $3: 1-7,2009$.

[11] K. Shimizu, M. Blajan, and T. Kuwabara. Removal of indoor air contaminant by atmospheric microplasma. Industry Applications, IEEE Transactions on, 47(6):2351 -2358, nov.-dec. 2011.

[12] R.H. Johnston, L.P. Ager, and J.G. McRory. A new small antenna efficiency measurement method. In Antennas and Propagation Society International Symposium, 1996. AP-S. Digest, volume 1, pages $176-179$ vol.1, jul 1996.

[13] H.A. Wheeler. The radiansphere around a small antenna. Proceedings of the IRE, 47(8):1325 -1331, aug. 1959.

[14] D. Agahi and W. Domino. Efficiency measurements of portable-handset antennas using the wheeler cap. Applied Microwave \& Wireless, 12:34-42, 2000.

[15] Robert L Merlino. Understanding langmuir probe current-voltage characteristics. American Journal of Physics, 75:1078, 2007.

[16] Michael A Lieberman and Allan J Lichtenberg. Principles of plasma discharges and materials processing. Cambridge Univ Press, 1994.

[17] G. Neumann, U. Banziger, M. Kammeyer, and M. Lange. Plasma-density measurements by microwave interferometry and langmuir probes in an rf discharge. Review of Scientific Instruments, 64(1):19-25, 1993.

[18] M Tuszewski and J A Tobin. The accuracy of langmuir probe ion density measurements in low-frequency rf discharges. Plasma Sources Science and Technology, 5(4):640, 1996.

[19] H. Sabadil, S. Klagge, and M. Kammeyer. Langmuir probe measurements of axial variation of plasma parameters in $27.1 \mathrm{mhz}$ rf oxygen planar discharges. Plasma Chemistry and Plasma Processing, 8(4):425-444, 1988.

[20] J. Hopwood, C. R. Guarnieri, S. J. Whitehair, and J. J. Cuomo. Langmuir probe measurements of a radio frequency induction plasma. Journal of Vacuum Science and Technology A: Vacuum, Surfaces, and Films, 11(1):152-156, 1993. 